\title{
Composición, estructura y flujo energético del meiobentos frente a Chile central
}

\author{
Composition, structure and energy flux of the meiobenthos off central Chile
}

\author{
JAVIER SELLANES ${ }^{1}$, CARLOS NEIRA ${ }^{2} \&$ EDUARDO QUIROGA ${ }^{3}$ \\ ${ }^{1}$ Centro de Investigación Oceanográfica en el Pacífico Sur-Oriental (COPAS), Universidad de Concepción, \\ Casilla 160-C, Concepción, Chile; e-mail: jsellane@udec.cl \\ ${ }^{2}$ Integrative Oceanography Division, Scripps Institution of Oceanography, 9500 Gilman Drive, La Jolla, \\ California 92093-0218, USA; e-mail: cneira@coast.ucsd.edu \\ ${ }^{3}$ Programa Doctorado Oceanografía, Universidad de Concepción, Casilla 160-C, \\ Concepción, Chile; e-mail: equiroga@udec.cl
}

\begin{abstract}
RESUMEN
Se estudió la estructura de la meiofauna metazoaria (a nivel de grandes grupos taxonómicos) y su rol en el flujo de energía en el subsistema bentónico frente a Concepción ( 3630'S). Las muestras se recolectaron en mayo y noviembre de 1997 y mayo de 1998 en cinco sitios correspondientes a: interior de la Bahía de Concepción $(28 \mathrm{~m})$, boca de la bahía $(35 \mathrm{~m})$, plataforma interior $(64 \mathrm{~m})$, plataforma intermedia $(88 \mathrm{~m})$ y plataforma externa $(120 \mathrm{~m})$. El periodo de estudio coincidió con el evento El Niño 1997-1998 (EN). El meiobentos resultó ser poco diverso a nivel de grandes grupos, pero con abundancias y biomasas moderadas a altas, en comparación con los valores promedio reportados en la literatura para el sublitoral fangoso. Nematoda fue el grupo dominante, con más de un $95 \%\left(10^{3}-10^{4}\right.$ ind $\left.10 \mathrm{~cm}^{-2}\right)$ de la densidad total, seguidos por Copepoda y Polychaeta. Las mayores abundancias y biomasas totales se encontraron siempre en la boca de la bahía, seguida de su parte interior y de la plataforma interior, mientras que los menores valores se ubicaron en la plataforma intermedia y externa.

Frente a Concepción el meiobentos juega un importante rol en el flujo de energía a través del subsistema bentónico. Se estimó que estos organismos podrían estar remineralizando y/o convirtiendo a biomasa hasta un 36 y $45 \%$ del carbono orgánico que llega desde la columna de agua a los sedimentos en el centro y en la boca de la bahía, respectivamente.
\end{abstract}

Palabras clave: meiofauna, producción secundaria, flujo energético, El Niño, Chile central.

\begin{abstract}
The general objective of this study was to determine the structure of metazoan meiofauna (at a high taxonomic level) and to estimate its role in the energy flux of the benthic sub-system off Concepción, Chile ( 36030' S). Samples were collected in May and November 1997 and May 1998 at five sites located at the: inner Bay of Concepción $(28 \mathrm{~m})$, bay-mouth $(35 \mathrm{~m})$, inner-shelf $(64 \mathrm{~m})$, mid-shelf $(88 \mathrm{~m})$ and outer-shelf $(120 \mathrm{~m})$. The study period coincided with the El Niño 1997-1998 (EN) event. The diversity of the meiobenthos was low, but density and biomass were moderate to high compared with average values reported for muddy sub-littoral environments elsewhere. Nematodes were the dominant group, with over 95 $\%\left(10^{3}-10^{4}\right.$ ind $\left.10 \mathrm{~cm}^{-2}\right)$ of the total density, followed by copepods and polychaetes. Highest densities and biomasses were always found at the bay-mouth, followed by the inner-bay and the inner-shelf, while the lowest values occurred at the mid- and outer-shelf. Off Concepción, the meiofauna plays an important role in the energy flux through the benthic sub-system. Indeed, the meiofauna could be converting to biomass and remineralizing in the inner-bay and bay-mouth up to 36 and $45 \%$, respectively, of the organic carbon reaching the sediments from the water column.
\end{abstract}

Key words: meiofauna, secondary production, energetic flux, El Niño, central Chile. 


\section{INTRODUCCIÓN}

La fauna bentónica es uno de los eslabones más importantes en el flujo de materia y energía en el ecosistema global. El destino del material orgánico generado por la producción primaria, que escapa a los procesos de degradación en la columna de agua y alcanza los sedimentos, depende de una serie de procesos bentónicos. Dentro de estos procesos, los más importantes son la remineralización y la incorporación a biomasa (Aller et al. 2002, Duineveld et al. 1997). De los distintos grupos de organismos bentónicos que participan en estos procesos, el estudio del rol de la meiofauna metazoaria sublitoral ha cobrado importancia en los últimos tiempos (Coull 1990, Giere 1993, Coull et al. 1995, 1999, Street et al. 1998), siendo considerada en la trama trófica bentónica el equivalente al zooplancton en el sistema pelágico. Asimismo, su actividad bioperturbadora, especialmente en las capas superficiales del sedimento, promueve la disgregación de estructuras biogénicas y liberación de carbono orgánico disuelto (Neira \& Höpner 1994), estimulando la producción bacteriana, la remineralización de la materia orgánica y la regeneración de nutrientes (Tenore 1977, Findlay \& Tenore 1982, Aller \& Aller 1992).

En general, en las zonas costeras la biomasa de la meiofauna es menor que la de la macrofauna. Sin embargo, debido a sus mayores tasas de renovación $(\mathrm{P} / \mathrm{B})$, el meiobentos cumple un rol proporcionalmente mayor en la transferencia de energía. Por consiguiente, los requerimientos metabólicos de la meiofauna (producción secundaria y respiración) podrían alcanzar valores incluso mayores a los registrados para la macrofauna, en particular en aquellos ecosistemas donde la razón de la biomasa macrofauna: meiofauna se aproxima o es inferior a 5:1 (Gerlach 1971, Higgins \& Thiel 1988).

En los últimos años se han realizado esfuerzos para entender la dinámica de las comunidades bentónicas en el subsistema bentónico frente a Chile central (Gutiérrez et al. 2000, Neira et al. 2001c), sin embargo aún se carece de evaluaciones cuantitativas de los requerimientos metabólicos que permitan realizar aproximaciones sobre los flujos de carbono orgánico en este ecosistema.

El ambiente prevaleciente sobre el subsistema bentónico frente a Chile central conjuga además una serie de características particulares, como bajos niveles de oxígeno (hipoxia; $<0,5 \mathrm{ml} \mathrm{L}^{-1}$ ), un alto flujo de materia orgánica a los sedimentos y altas tasas de remineraliza- ción (Gutiérrez et al. 2000). En adición, las comunidades macrobentónicas muestran una baja riqueza de especies, dominadas ocasionalmente por bacterias filamentosas gigantes (Gallardo et al. 1995). A pesar de la potencial importancia tanto evolutiva como funcional que el meiobentos podría tener bajo estas condiciones, su ecología recién está comenzando a ser estudiada en el margen del Pacífico suroriental (Rowe 1985, Neira et al. 2001a, 2001b, 2001c).

En el presente estudio se evaluó el rol que la meiofauna metazoaria podría tener en el flujo de energía a través del sistema bentónico frente a Chile central. Para ello, se estimó su producción secundaria, su respiración en términos de carbono y se evaluó su importancia en el ecosistema a través de una comparación con la macrofauna y con valores del flujo de $\mathrm{C}_{\text {org }}$ a los sedimentos indicados para la zona. Asimismo, los resultados obtenidos fueron comparados con valores reportados para otros ecosistema de similares condiciones tróficas.

Por otra parte, cabe destacar que este estudio coincidió con El Niño 1997-1998 (EN), el cual tuvo un efecto general positivo sobre la abundancia, biomasa y diversidad de las comunidades macro y meiobentónicas locales (Gutiérrez et al. 2000, Neira et al. 2001c). Por consiguiente, el presente trabajo complementa los resultados reportados previamente y entrega nuevos antecedentes sobre las repercusiones que este evento podría tener sobre el flujo de energía mediado por las comunidades bentónicas.

\section{MATERIALES Y MÉTODOS}

Los muestreos fueron realizados en mayo y noviembre de 1997 y mayo de 1998, coincidiendo con el EN 1997-1998, considerado uno de los eventos cálidos más severos registrados hasta la fecha (Kane 1999, McPhaden 1999). El área de estudio comprendió cinco sitios de muestreo ubicados a lo largo de una transecta (Fig. 1); el interior de la bahía de Concepción (Estación 4, $28 \mathrm{~m}$ ), la boca de la bahía (Estación 7, $35 \mathrm{~m}$ ), la plataforma interna (Estación 14, $64 \mathrm{~m}$ ), la plataforma intermedia (Estación 18, $88 \mathrm{~m}$ ) y el borde de la plataforma continental adyacente (Estación 26, $120 \mathrm{~m}$ ). Las muestras se recolectaron a bordo de la embarcación L/C "Kay Kay" (Universidad de Concepción) y se utilizó como base de operaciones la Estación de Biología Marina de Dichato. En mayo de 1997, las muestras de sedimentos fueron tomadas utilizando un mono-sacatestigos tipo Rumohr (diámetro interno 7,5 cm). En las demás oportunidades se utilizó un multi-sacatestigos, con un 


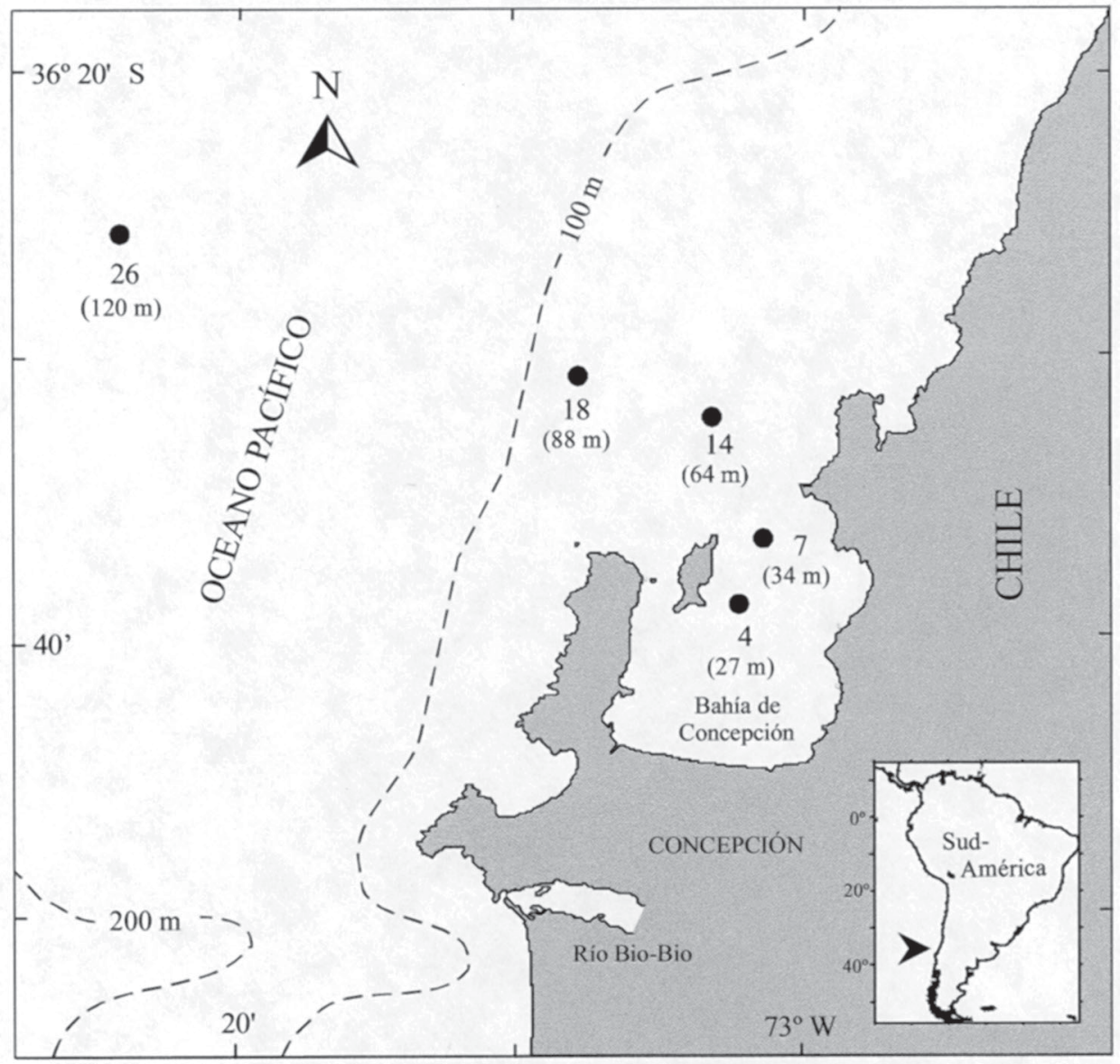

Fig. 1: Localización de las cinco estaciones de muestreo: Est. 4, centro de la Bahía de Concepción; Est. 7, boca de la bahía; Est 14, plataforma continental interior; Est. 18, plataforma intermedia y 26, plataforma exterior.

Location of the five sampling sites: Est. 4, inner Bay of Concepción; Est. 7, bay-mouth; Est. 14, inner-shelf; Est. 18, midshelf and Est. 26, outer-shelf.

principio de operación similar al descrito por Barnett et al. (1984) y dotado de seis tubos saca-testigos de $9 \mathrm{~cm}$ de diámetro interno y 100 $\mathrm{cm}$ de largo. La profundidad de penetración en el sedimento siempre fue de al menos $20 \mathrm{~cm}$ y solo se utilizaron los testigos que no presentaron evidencias de resuspensión o alteraciones del sedimento durante el procedimiento de muestreo.

\section{Análisis de la meiofauna}

En cada estación se recolectaron tres muestras, cada una proveniente de lances distintos, mediante subtestigos de plexiglás de $3,6 \mathrm{~cm}$ de diámetro interno $\left(10 \mathrm{~cm}^{2}\right.$ de área interna). En el laboratorio, se procesaron los $15 \mathrm{~cm}$ superficiales de cada subtestigo, los cuales para facilitar su posterior manipulación fueron seccionados verticalmente en cuatro fracciones $(0-2,2-5,5$ 10 y $10-15 \mathrm{~cm})$. Las abundancias reportadas en este estudio corresponden entonces a los $15 \mathrm{~cm}$ superficiales y a su vez promediadas de las tres réplicas tomadas en cada sitio.

Antes de fijar las muestras, estas se trataron con una solución de $\mathrm{MgCl}_{2}$ al $6 \%$ por $15 \mathrm{~min}$. Este compuesto tiene un efecto anestésico, que contribuye a la liberación de organismos que puedan estar adheridos a las partículas de sedimento (Pfannkuche \& Thiel 1988). Se fijó con formalina al $4 \%$ tamponada con bórax. El tamizado $(0,063-0,5 \mathrm{~mm})$ se realizó aplicando la metodología de resuspensión-decantación (Wieser 1960) después de sonicar el sedimento por $10 \mathrm{~s}$ (Thiel et al. 1975). Se ha demostrado que este procedimiento es sumamente eficiente en sedimentos fangosos y arcillosos (Murrell \& Fleeger 1989), sustrato predominante en el área 
de estudio. La eficiencia de extracción se comprobó inspeccionando ocasionalmente el sedimento remanente, resultando cercana al $100 \%$ para organismos sin tests calcáreos (i.e., mayor parte de la meiofauna metazoaria permanente). Los organismos así obtenidos fueron preservados en formalina al $4 \%$ con Rosa de Bengala hasta su posterior conteo e identificación bajo lupa estereoscópica. La biomasa de los distintos grupos se derivó de la fórmula de Andrassy (1956):

$$
V=C * \mathrm{~L} * W^{2}
$$

donde $V$ es el volumen en $\mathrm{mL}, C$ es una constante de acuerdo a cada taxón, L es el largo del organismo y $W$ el ancho, estas dos últimas medidas son en mm. Los valores de la constante $C$ se obtuvieron de Feller \& Warwick (1988). El volumen se convirtió a peso húmedo, multiplicándolo por la gravedad específica del organismo $\left(1,13 \mathrm{~g} \mathrm{~cm}^{-3}\right)$.

Los valores de la biomasa de la macrofauna $(>0,5 \mathrm{~mm})$ para los correspondientes sitios y meses de muestreo se tomaron de Gutiérrez et al. (2000). En breve, las muestras para macrofauna en cada oportunidad se recolectaron de 4 testigos provenientes de lances independientes, de los cuales se analizaron los $20 \mathrm{~cm}$ superficiales.

La producción secundaria $(P)$ de la meiofauna se estimó utilizando el modelo empírico de Tumbiolo \& Downing (1994) para invertebrados marinos:

$$
\begin{gathered}
\log P=0,18+0,97 \log B-0,22 \log W_{m}+ \\
0,04 T_{f}-0,014 T_{f} \log (Z+1)
\end{gathered}
$$

donde $P$ es la producción en $\mathrm{g}$ (peso seco) $\mathrm{m}^{-2}$ $\mathrm{a}^{-1}, B$ es la biomasa en mg peso seco, $W_{m}$ es la máxima masa corporal en mg peso seco, $T_{f}$ es la temperatura del agua de fondo y $Z$ es la profundidad en $m$.

La respiración total de la meiofauna $\left(R_{t}\right)$ se estimó a través de la ecuación propuesta por Schwinghamer et al. (1986):

$$
\log _{10} R_{t}=0.367+0.993 \log _{10} P
$$

donde $R t$ es la respiración total y $P$ es la producción de la meiofauna en kilocalorías [kcal $\mathrm{m}^{-2} \mathrm{a}^{-1}$ (Banse \& Mosher 1980)]. El cociente de respiración (CR) utilizado para el cálculo de la demanda de oxígeno de la meiofauna fue de 0,85 $\left(\mathrm{CR}=\right.$ moles $\mathrm{CO}_{2} /$ moles $\left.\mathrm{O}_{2}=0,85\right)$. Los valores de biomasa, producción y respiración fueron expresados en gramos de carbono ( $\mathrm{g} \mathrm{C}$ $\mathrm{m}^{-2}$ ) de acuerdo a los factores de conversión propuestos por Brey (2001).

\section{Análisis estadístico de los datos}

Los cambios espaciales y temporales en la abundancia total y por grupos fueron evaluados mediante análisis de varianza (ANDEVA) de dos vías $(\mathrm{a}=0,05)$, tomando las estaciones como factores fijos y los meses como aleatorios. Se trabajó con datos transformados aplicando la raíz cuadrada y en todos los casos se verificó la condición de homogeneidad de varianza (prueba de Bartlett) así como la normalidad de los residuos (Zar 1974). En caso de encontrarse diferencias significativas, los contrastes se efectuaron mediante la prueba a posteriori HSD de Tukey. Para analizar la similitud entre sitios tanto espacial como temporal, se realizó además un análisis de conglomerado en base a las abundancias de los grupos más característicos. Se incluyeron todos los sitios y fechas de recolección de muestras y se utilizó el método de aglomeración UPGMA ("Unweighted pair-group average", Sneath \& Sokal 1973) con base en la matriz de similitud de Bray-Curtis. Los análisis se efectuaron utilizando los programas estadísticos Statistica 5.1 (StatSoft Inc., StatSoft, Tulsa, Oklahoma, USA) y Primer 5.1.2 (Primer-E Ltd.).

\section{RESULTADOS}

\section{Composición taxonómica y abundancia}

En la Tabla 1 se presenta la contribución porcentual de cada taxón a la abundancia total. Los nemátodos constituyeron siempre más del 95\% en todas las estaciones. Otros grupos como los poliquetos, incluyendo juveniles de especies macrobentónicas (meiofauna temporal) y los crustáceos, principalmente copépodos harpacticoídeos, no superaron el $2 \%$ de las abundancias totales. La contribución porcentual de los restantes 12 taxa mayores identificados nunca superó el $0,5 \%$. Los nemátodos dictaron entonces el patrón general de la abundancia total de la meiofauna en todos los sitios y muestreos (Fig. 2A y 2E) $\left(\mathrm{r}^{2}=0,99 ; \mathrm{n}=45, \mathrm{P}<0,001\right)$. Estos organismos alcanzaron densidades máximas de $10.360 \pm 1.984$ ind $10 \mathrm{~cm}^{-2}$ (media \pm EE) en la boca de la bahía (mayo de 1997), mientras que el mínimo se ubicó en el sitio más profundo, $757 \pm 204$ ind $10 \mathrm{~cm}^{-2}$ en mayo de 1998 (Fig. 2A). Las abundancias de los demás grupos fueron siempre al menos un orden de magnitud inferiores, encontrándose también las mayores abundancias de grupos como Copepoda y Polychaeta en la boca de la bahía y los menores en la plataforma media y exterior. En 


\section{TABLA 1}

Abundancia relativa (como porcentaje del total) de los grupos mayores de la meiofauna para todo el período de estudio

Relative abundance (\%) of major meiofaunal groups during the study period

\begin{tabular}{|c|c|c|c|c|c|c|c|c|c|c|c|c|c|c|c|c|c|}
\hline Estación & Nem & Cop & $\mathrm{Nau}$ & Pol & Ost & Hal & Rot & Biv & Amp & Tar & Cum & Tur & Gas & Gsp & Oli & Kin & Otros \\
\hline \multicolumn{18}{|c|}{ Mayo 1997} \\
\hline 499,3 & 0,23 & 0,14 & 0,32 & - & - & - & $*$ & $*$ & - & - & - & - & - & - & - & - & \\
\hline 795,9 & 1,28 & 1,34 & 1,06 & 0,27 & 0,03 & 0,02 & $*$ & - & $*$ & - & - & $*$ & - & - & $*$ & - & \\
\hline 14 & 98,1 & 0,60 & 0,25 & 0,98 & - & 0,02 & 0,01 & - & 0,06 & - & - & - & - & - & - & - & - \\
\hline 18 & 99,6 & - & - & 0,37 & - & 0,01 & - & 0,01 & - & - & - & - & 0,01 & - & - & - & - \\
\hline 26 & 99,3 & 0,05 & 0,08 & 0,05 & - & 0,17 & 0,09 & - & - & 0,07 & 0,07 & - & - & - & - & - & - \\
\hline \multicolumn{18}{|c|}{ Noviembre 1997} \\
\hline 498,0 & 1,22 & 0,27 & 0,42 & - & 0,02 & 0,08 & 0,01 & - & 0,01 & 0,01 & - & - & - & - & - & 0,01 & \\
\hline 799,0 & 0,48 & 0,09 & 0,25 & 0,12 & - & 0,01 & - & - & - & $*$ & - & - & - & - & - & - & \\
\hline 14 & 99,2 & 0,46 & 0,06 & 0,29 & 0,01 & 0,01 & - & - & - & 0,01 & - & - & - & - & - & - & - \\
\hline 18 & 98,3 & 1,02 & 0,16 & 0,32 & - & 0,02 & 0,08 & - & - & - & - & - & - & - & - & - & 0,06 \\
\hline 26 & 97,9 & 1,40 & 0,29 & 0,20 & - & 0,09 & 0,09 & 0,03 & - & - & - & - & - & - & - & - & - \\
\hline \multicolumn{18}{|c|}{ Mayo 1998} \\
\hline 498,6 & 0,27 & 0,61 & 0,53 & 0,01 & - & - & - & 0,01 & - & - & - & - & - & - & - & - & \\
\hline 798,3 & 0,61 & 0,73 & 0,25 & 0,04 & $*$ & - & - & 0,02 & - & - & - & - & - & - & - & - & \\
\hline 14 & 98,5 & 0,56 & 0,79 & 0,20 & 0,20 & - & - & - & - & - & - & - & - & - & - & - & - \\
\hline 18 & 98,5 & 0,93 & 0,14 & 0,37 & - & - & - & - & - & - & 0,07 & - & - & - & - & - & - \\
\hline 26 & 97,6 & 1,59 & 0,75 & 0,04 & - & - & - & 0,04 & - & - & - & - & - & - & - & - & - \\
\hline
\end{tabular}

Clave de abreviaciones: Nem= Nematoda, Cop= Copepoda, Naup= Nauplii, Pol= Polychaeta, Ost $=$ Ostracoda, Hal $=$ Halacarida, Rot $=$ Rotifera, Biv $=$ Bivalvia, Amp $=$ Amphipoda, Tar $=$ Tardigrada, Cum $=$ Cumacea, Tur $=$ Turbellaria, Gas $=$ Gastrotticha, Gsp= Gastropoda, Oli= Oligochaeta, Kin= Kinorhyncha

$(-) ;(*)$ presente pero en porcentaje inferior a 0,01

mayo de 1997, copépodos (adultos) y poliquetos alcanzaron en la boca de la bahía, abundancias de $283 \pm 83$ y $114 \pm 27$ ind $10 \mathrm{~cm}^{-2}$, respectivamente (Fig. 2B y 2C). En la plataforma media (Estación 18) al comienzo del período no se registraron copépodos, mientras que los poliquetos fueron más escasos $\left(<1\right.$ ind $\left.10 \mathrm{~cm}^{-2}\right)$ en la plataforma externa (Estación 26). Otros grupos como Turbellaria, Gastrotricha, Rotifera, Kinorhyncha, Oligochaeta, Tardigrada, Amphipoda, Cumacea, Ostracoda, Halacaroidea, Gastropoda y Bivalvia, en general, fueron representados por pocos ejemplares y solo ocasionalmente, con densidades en torno a 10 ind $10 \mathrm{~cm}^{-2}$. La única excepción fue la boca de la bahía (Estación 7) en mayo de 1997, con 38 \pm 18 ostrácodos $10 \mathrm{~cm}^{-2}$, los que contribuyeron con un $0,27 \%$ a la abundancia total (Tabla 1 ).

\section{Variación temporal y espacial}

Si bien no se observó diferencias significativas entre las abundancias totales globales para los tres meses de muestreo $\left(\mathrm{F}_{2,30}=1,35 ; \mathrm{P}=\right.$ $0,270)$, se detectó una fuerte interacción entre sitios y meses $\left(\mathrm{F}_{8,30}=3,93 ; \mathrm{P}=0,003\right)$. En este sentido, a pesar de la falta de consistencia tem- poral entre las estaciones, lo más destacable fue el significativo aumento en la abundancia total en la estación al interior de la bahía (ANDEVA y prueba de Tukey). En la plataforma interior (Estación 14) si bien las diferencias no fueron significativas entre los tres meses, también se observó un sostenido aumento que produjo que en mayo de 1998 las abundancias en este sitio se diferenciaran significativamente de las de plataforma externa. En cuanto a los copépodos adultos y los nauplios, en general se observó una tendencia al aumento en las abundancias principalmente en el interior de la bahía y en menor grado en las dos estaciones más profundas. Además, la abundancia promedio de nauplios en mayo de 1998 fue significativamente mayor $\left(\mathrm{F}_{2,30}=12,87 ; \mathrm{P}<0,001\right)$.

Exceptuando el interior de la bahía, los poliquetos mostraron una tendencia general hacia una disminución en sus abundancias hacia mayo de 1998, la cual fue más acentuada en la boca de la bahía y plataforma interna.

Un análisis de conglomerado basado en las abundancias de los grupos principales comparando los distintos sitios y períodos (similitud de Bray-Curtis), reveló dos grupos principales (Fig. 3 ), uno conformado principalmente por las estaciones ubicadas en la plataforma externa (Esta- 

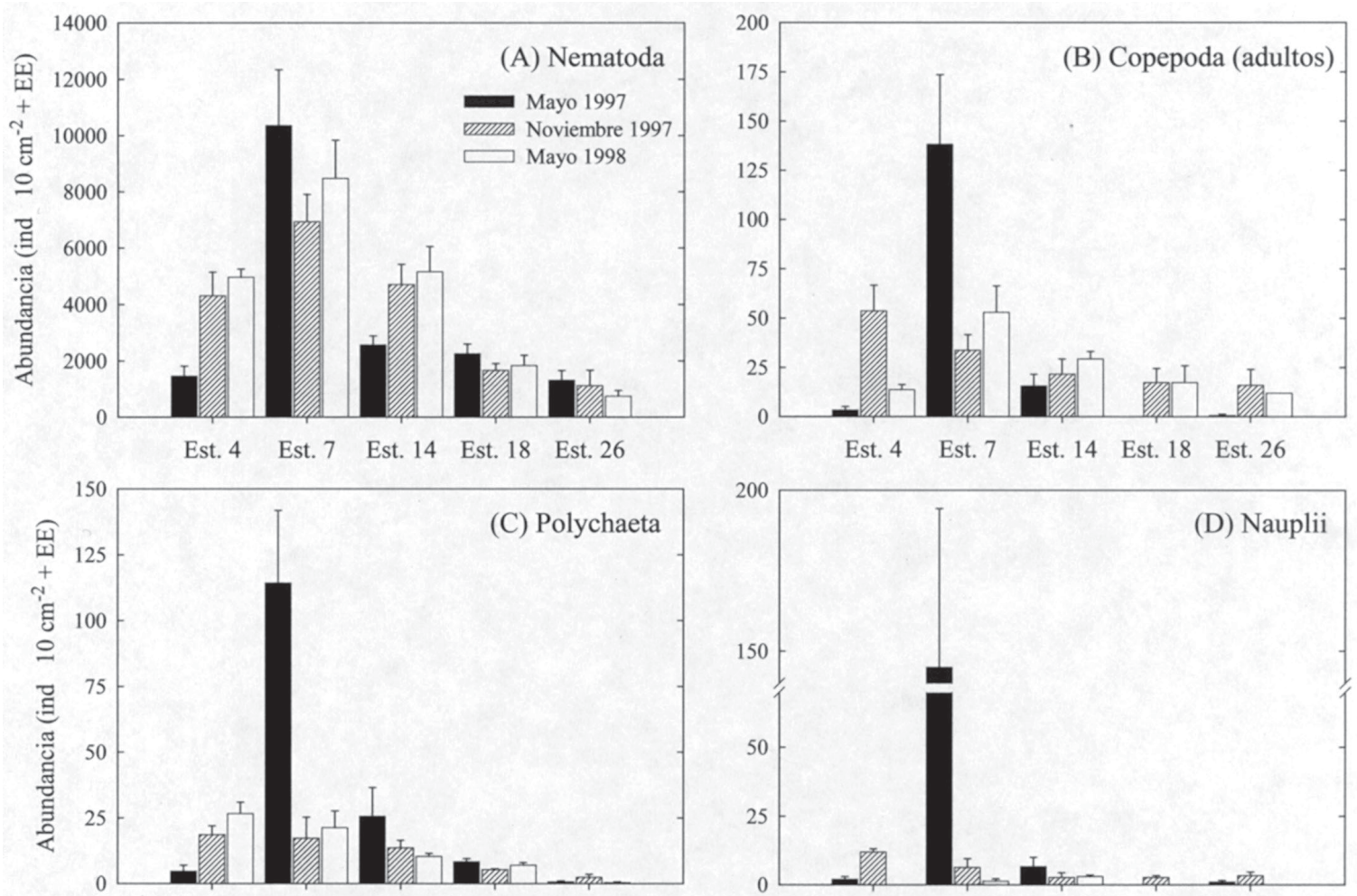

$\begin{array}{lllll}\text { Est. } 4 & \text { Est. } 7 & \text { Est. } 14 & \text { Est. } 18 & \text { Est. } 26\end{array}$

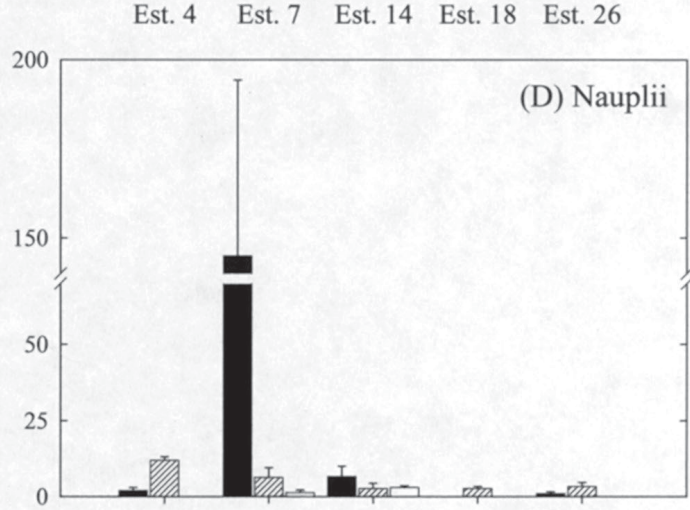

$\begin{array}{llllll}\text { Est. } 4 & \text { Est. } 7 & \text { Est. } 14 & \text { Est. } 18 & \text { Est. } 26\end{array}$

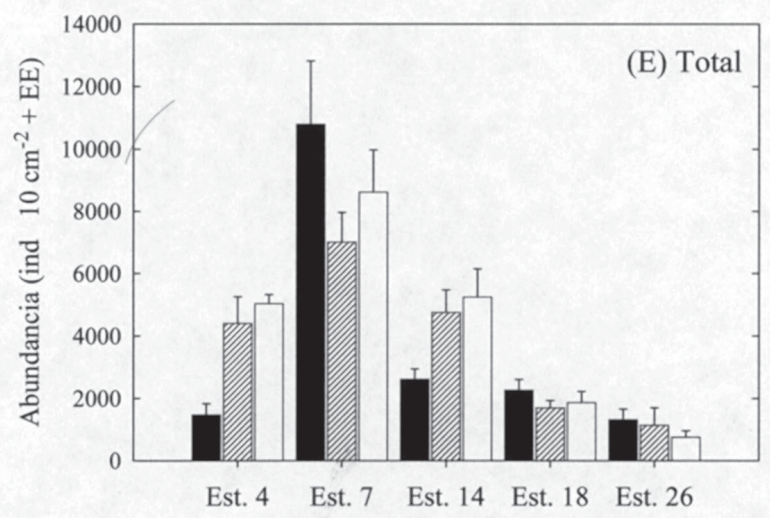

Fig. 2: Abundancia de la meiofauna por sitio y para cada muestreo: (A) Nematoda, (B) Copepoda (adultos), (C) Polychaeta, (D) Nauplii y (E) Total.

Meiofaunal abundance at each site and sampling date: (A) Nematoda, (B) Copepoda (adults), (C) Polychaeta, (D) Nauplii, and (E) Total.

ción 26) e intermedia (Estación 18) en todas las ocasiones junto con el centro de la bahía (Estación 3) y plataforma interna (Estación 14) al comienzo del estudio (mayo de 1997) y caracterizado por bajas abundancias totales. El segundo grupo, caracterizado por mayores abundancias, estuvo constituido por la boca de la bahía (Estación 7) durante todo el estudio junto al interior de la bahía (Estación 4) y plataforma interna (Estación 14) hacia mayo de 1998.
Biomasa, estimación de la producción secundaria y respiración

El sitio donde la meiofauna presentó siempre la mayor biomasa (Tabla 2) fue la boca de la bahía $\left(17,73 \mathrm{~g} \mathrm{~m}^{-2}\right.$ en promedio) mientras que los menores valores se registraron en la plataforma externa $\left(\sim 2 \mathrm{~g} \mathrm{~m}^{-2}\right)$. Al igual que con la abundancia, los nemátodos contribuyeron en más de un $90 \%$ a los valores de la biomasa total en todos los sitios. 


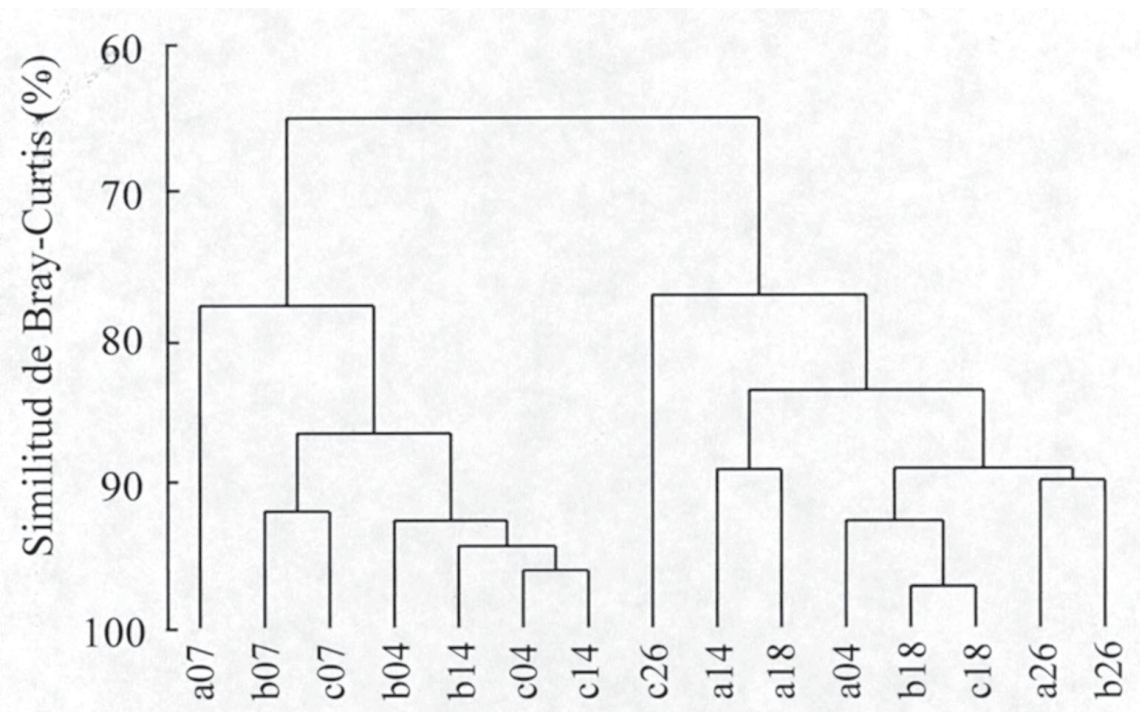

Fig. 3: Agrupamiento por sitios y muestreo en base al índice de similitud de Bray-Curtis a partir de la matriz de abundancia por grupos taxonómicos mayores y utilizando el método de agrupación UPGMA: (a) = mayo 1997, (b) = noviembre 1997 y (c) = mayo 1998. Ejemplo: b07 = Est. 7, noviembre de 1997.

Clustering by site and sampling date based in Bray-Curtis similarity index, calculated from the abundance matrix by major taxonomic groups and using the UPGMA joining method: (a) = May 1997, (b) = November 1997, (c) = May 1998. Example: b07 = Est. 7, November 1997.

\section{TABLA 2}

Biomasa de la meiofauna (media \pm EE) para los meses de mayo y noviembre de 1997 y mayo de 1998

Meiofaunal biomass (mean \pm SE) during May and November 1997 and May 1998

\begin{tabular}{|c|c|c|c|c|c|}
\hline \multirow[t]{2}{*}{ Estación } & \multicolumn{5}{|c|}{ Biomasa húmeda $\left(\mathrm{g} \mathrm{m}^{-2}\right)$} \\
\hline & Nematoda & Copepoda & Polychaeta & Otros & Total \\
\hline \multicolumn{6}{|c|}{ Mayo 1997} \\
\hline 4 & $2,63 \pm 0,63$ & * & $0,05 \pm 0,02$ & * & $2,70 \pm 0,65$ \\
\hline 7 & $18,57 \pm 3,55$ & $0,54 \pm 0,16$ & $1,2 \pm 0,28$ & $0,07 \pm 0,03$ & $20,36 \pm 3,80$ \\
\hline 14 & $4,61 \pm 0,58$ & $0,06 \pm 0,02$ & $0,27 \pm 0,11$ & $0,05 \pm 0,00$ & $4,97 \pm 0,63$ \\
\hline 18 & $4,05 \pm 0,61$ & - & $0,09 \pm 0,01$ & $*$ & $4,14 \pm 0,62$ \\
\hline 26 & $2,35 \pm 0,62$ & $*$ & $*$ & $*$ & $2,38 \pm 0,62$ \\
\hline \multicolumn{6}{|c|}{ Noviembre 1997} \\
\hline 4 & $7,73 \pm 1,51$ & $0,14 \pm 0,03$ & $0,19 \pm 0,03$ & $0,08 \pm 0,04$ & $8,12 \pm 1,57$ \\
\hline 7 & $13,72 \pm 1,92$ & $0,08 \pm 0,01$ & $0,19 \pm 0,08$ & $0,05 \pm 0,01$ & $14,02 \pm 1,92$ \\
\hline 14 & $8,45 \pm 1,29$ & $0,06 \pm 0,02$ & $0,15 \pm 0,03$ & $*$ & $8,65 \pm 1,32$ \\
\hline 18 & $2,99 \pm 0,43$ & $0,06 \pm 0,02$ & $0,06 \pm 0,00$ & $*$ & $3,11 \pm 0,43$ \\
\hline 26 & $2,00 \pm 0,99$ & $*$ & $*$ & $*$ & $2,07 \pm 1,01$ \\
\hline \multicolumn{6}{|c|}{ Mayo 1998} \\
\hline 4 & $8,9 \pm 0,52$ & $0,08 \pm 0,01$ & $0,27 \pm 0,04$ & $*$ & $9,25 \pm 0,53$ \\
\hline 7 & $18,35 \pm 2,94$ & $0,21 \pm 0,01$ & $0,23 \pm 0,06$ & $*$ & $18,82 \pm 2,95$ \\
\hline 14 & $9,25 \pm 1,60$ & $0,12 \pm 0,01$ & $0,11 \pm 0,01$ & - & $9,48 \pm 1,63$ \\
\hline 18 & $3,30 \pm 0,64$ & $0,06 \pm 0,02$ & $0,08 \pm 0,01$ & $*$ & $3,44 \pm 0,66$ \\
\hline 26 & $1,33 \pm 0,36$ & $0,05 \pm 0,00$ & $*$ & $*$ & $1,38 \pm 0,37$ \\
\hline
\end{tabular}

(*) Presente pero con biomasa inferior a $0,01 \mathrm{~g} \mathrm{~m}^{-2}$

(-) Ausente 
Los valores de producción secundaria estimados fluctuaron entre $1,53 \mathrm{~g} \mathrm{C} \mathrm{m}^{-2} \mathrm{a}^{-1}$ para el área de la plataforma externa (Estación 26) en mayo 1998 a 32,2 $\mathrm{g} \mathrm{C} \mathrm{m}^{-2} \mathrm{a}^{-1}$ para la boca de la bahía (Estación 7) en mayo 1997 (Tabla 3). Las tendencias tanto espaciales como temporales fueron similares a las de la abundancia. Las razones producción: biomasa obtenidas $(\mathrm{P} / \mathrm{B}) \mathrm{o}$ tasas de renovación variaron entre 10 y $18 \mathrm{a}^{-1}$. En general las mayores tasas se encontraron en los sitios más someros. Las tasas registradas en noviembre (1997) fueron levemente inferiores a las correspondientes a mayo de 1997 y 1998 (Tabla 3). Los valores de respiración, estimados a través de la ecuación propuesta por Schwinghamer et al. (1986), presentaron un patrón similar a lo encontrado para la producción. Estos variaron entre $3,50 \mathrm{~g} \mathrm{C} \mathrm{m}^{-2} \mathrm{a}^{-1}$ en la plataforma externa (Estación 26) en mayo 1998 y $72,09 \mathrm{~g} \mathrm{C} \mathrm{m}^{-2} \mathrm{a}^{-1}$ en la boca de la bahía (Estación 7) en mayo 1997 (Tabla 3).

En tanto, la producción + respiración en el área de estudio registró valores que fluctuaron entre 15,66 y 104,28 $\mathrm{g} \mathrm{C} \mathrm{m}^{-2} \mathrm{a}^{-1}$ en las áreas más someras, mientras que en la zona de la plataforma media y externa fluctuaron entre 5,03 y $15,60 \mathrm{~g} \mathrm{C} \mathrm{m}^{-2} \mathrm{a}^{-1}$ (Tabla 3).

\section{DISCUSIÓN}

Comparación con otros ambientes costeros y áreas de surgencia

Las densidades promedio registradas en este estudio fueron comparables e incluso superiores a las de otras áreas con características similares a las encontradas frente a Concepción (Tabla 4). Sin embargo, valores como los observados en la Estación 7 ( $>10^{4}$ ind $10 \mathrm{~cm}^{-2}$ ) (Fig. 2E), se encuentran entre los más altos registrados para ambientes marinos sublitorales, siendo solo comparables a aquellos de marismas e intermareal fangoso (Coull 1988, de Bovée 1996). En tanto, los sitios ubicados sobre la plataforma intermedia y exterior registraron abundancias promedio similares a lo descrito para ambientes fangosos sublitorales [1.000-2.000 ind $10 \mathrm{~cm}^{-2}$ (de Bovée et al. 1996)]. Si se tiene en cuenta que en los sitios sobre la plataforma intermedia y externa (estaciones 18 y 26) las aguas ecuatoriales subsuperficiales (AESS) predominan durante todo el año y por lo tanto prevalecen las condiciones deficientes en oxígeno (Gallardo 1985), serían esperables densidades aún menores. A modo de comparación, densidades de

TABLA 3

Resumen de resultados de la biomasa total $(\mathrm{B})$, producción secundaria $(\mathrm{P})$, productividad $(\mathrm{P} / \mathrm{B})$ y respiración total meiobentónica $\left(\mathrm{R}_{\mathrm{t}}\right)$ en el área de estudio

Summary of results of total biomass $(B)$, secondary production $(\mathrm{P})$, productivity $(\mathrm{P} / \mathrm{B})$ and total meiobenthic respiration $\left(\mathrm{R}_{\mathrm{t}}\right)$ at the study area

\begin{tabular}{|c|c|c|c|c|c|}
\hline Estación & $\begin{array}{c}\mathrm{B} \\
\left(\mathrm{g} \mathrm{C} \mathrm{m}^{-2}\right)\end{array}$ & $\begin{array}{c}\mathrm{P} \\
\left(\mathrm{g} \mathrm{C} \mathrm{m}^{-2} \mathrm{a}^{-1}\right)\end{array}$ & $\begin{array}{l}\mathrm{P} / \mathrm{B} \\
\left(\mathrm{a}^{-1}\right)\end{array}$ & $\begin{array}{c}\mathrm{R} \\
\left(\mathrm{g} \mathrm{C} \mathrm{m}^{-2} \mathrm{a}^{-1}\right)\end{array}$ & $\begin{array}{c}\mathrm{P}+\mathrm{R} \\
\left(\mathrm{g} \mathrm{C} \mathrm{m}^{-2} \mathrm{a}^{-1}\right)\end{array}$ \\
\hline \multicolumn{6}{|c|}{ Mayo 1997} \\
\hline 4 & 0.27 & 4.79 & 18 & 10.87 & 15.66 \\
\hline 7 & 2.04 & 32.19 & 16 & 72.09 & 104.28 \\
\hline 14 & 0.50 & 7.26 & 15 & 16.43 & 23.69 \\
\hline 18 & 0.41 & 4.77 & 12 & 10.83 & 15.60 \\
\hline 26 & 0.24 & 2.67 & 11 & 6.08 & 8.75 \\
\hline \multicolumn{6}{|c|}{ Noviembre 1997} \\
\hline 4 & 0.81 & 12.71 & 16 & 28.65 & 41.36 \\
\hline 7 & 1.40 & 20.17 & 14 & 45.32 & 65.49 \\
\hline 14 & 0.87 & 9.96 & 12 & 22.49 & 32.45 \\
\hline 18 & 0.31 & 3.43 & 11 & 7.80 & 11.23 \\
\hline 26 & 0.21 & 2.10 & 10 & 4.79 & 6.89 \\
\hline \multicolumn{6}{|c|}{ Mayo 1998} \\
\hline 4 & 0.93 & 16.20 & 18 & 36.45 & 52.65 \\
\hline 7 & 1.88 & 30.52 & 16 & 68.37 & 98.89 \\
\hline 14 & 0.95 & 12.36 & 13 & 27.86 & 40.22 \\
\hline 18 & 0.34 & 4.01 & 12 & 9.11 & 13.12 \\
\hline 26 & 0.14 & 1.53 & 11 & 3.50 & 5.03 \\
\hline
\end{tabular}


TABLA 4

Comparación de las abundancias de la meiofauna en estudios realizados en zonas con características similares a la Bahía de Concepción y/o plataforma continental adyacente. Abundancias expresadas en ind $10 \mathrm{~cm}^{-2}$

Comparison of meiofaunal abundances among areas sharing similar characteristics with Concepción Bay and/or adjacent continental shelf. Abundances reported in ind $10 \mathrm{~cm}^{-2}$

\begin{tabular}{|c|c|c|c|c|c|c|}
\hline Lugar & $\begin{array}{r}\text { Profundidad } \\
(\mathrm{m})\end{array}$ & Características & Nemátodos & Copépodos & Total & Referencia \\
\hline \multicolumn{7}{|l|}{ Fiordos } \\
\hline Gullmar Fjord, Suecia & 115 & Anoxia estacional & $321-5415$ & $4-83$ & $326-5.688$ & Josefson \& Widbom (1988) \\
\hline \multicolumn{7}{|l|}{ Pisciculturas } \\
\hline Mar Tirreno, Italia & 10 & Debajo de cultivo de salmones $\mathrm{H}$ & Hasta $75 \%$ total & Hasta $15 \%$ total & $870-2.238$ & Mirto et al. (2000) \\
\hline Bahía de Fundy, Canadá & $12-14$ & Debajo de cultivo de salmones & $>88 \%$ & - & $2.307 \pm 573$ & Duplisea \& Hargrave (1996) \\
\hline \multicolumn{7}{|l|}{ Bahías eutroficadas } \\
\hline Bahía de St. Brieuc, Francia & $2,6-11$ & Orgánicamente enriquecida & $542-2.086$ & $34-611$ & $745-2.207$ & Le Guellec \& Bodin (1992) \\
\hline \multicolumn{7}{|l|}{$\begin{array}{l}\text { Zonas de surgencia y/0 } \\
\text { con alta carga orgánica }\end{array}$} \\
\hline Plataforma de Louisiana, USA & $28-106$ & Hipoxia estacional moderada & $566-5.859$ & $10-1865$ & $1.173-6.960$ & Radziejewska et al. (1996) \\
\hline Plataforma de Perú $\left(15^{\circ} \mathrm{S}\right)$ & $63-110$ & Borde superior de la ZMO & - & - & $609-1.134$ & Rowe (1975) \\
\hline Talúd de Perú $\left(12^{\circ} \mathrm{S}\right)$ & 305 & Centro de la ZMO & $1.502 \pm 430$ & 0 & $1.517 \pm 431$ & Neira et al. (2001b) \\
\hline NW de Africa & 25 & Surgencia costera & - & - & ca. 1.000 & Rowe (1975) \\
\hline Mar de Arabia & 400 & Surgencia estacional & ca. 1.700 & - & & Cook et al. (2000) \\
\hline Bahía de Concepción, Chile (centro, Est. 4) & 27 & Hipoxia severa/anoxia estacional & $1.463-4.964$ & $5-65$ & $1.473-5.036$ & Este estudio \\
\hline Bahía de Concepción, Chile (boca, Est. 7) & 34 & Hipoxia estacional & $4.738-10.360$ & $40-283$ & $4.855-10.796$ & Este estudio \\
\hline Plataforma interna, Chile (Est. 14) & 64 & Hipoxia estacional, borde ZMO & $2.106-5.896$ & $22-70$ & $2.170-5.989$ & Este estudio \\
\hline Plataforma intermedia, Chile (Est. 18) & 88 & Borde superior ZMO & 1.193-2.417 & $0-20$ & $1.202-2.433$ & Este estudio \\
\hline Plataforma externa, Chile (Est. 26) & 120 & Borde superior ZMO & $738-1.268$ & $1-31$ & $895-1.318$ & Este estudio \\
\hline
\end{tabular}

solo 25 a 150 ind $10 \mathrm{~cm}^{-2}$ fueron reportadas en áreas sublitorales deficientes en oxígeno de Suecia (Nyholm \& Olson 1973).

Los valores de biomasa total se ubicaron en el rango de los registrados por Rowe (1985) para el área de surgencia frente a Perú central $\left(\sim 3,8 \mathrm{~g} \mathrm{~m}^{-2}\right)$ y en general superior a valores reportados para áreas más oligotróficas.

La baja riqueza a nivel de grandes taxa, debido al dominio de los nemátodos tanto en abundancia (> $95 \%$ ) (Tabla 1) como en biomasa $(>91 \%)$ (Tabla 2$)$, comprueba que este grupo es particularmente exitoso en sedimentos orgánicamente ricos y deficientes en oxígeno (Riemann 1988, Cook et al. 2000, Neira et al. 2001b). Mientras que bajo estas condiciones ambientales, grupos como los crustáceos quedan excluidos o muy reducidos (Wells 1988, Neira et al. 2001b). La combinación de baja riqueza de taxa y alta abundancia de la meiofauna metazoaria en zonas de mínimo oxígeno está bien documentada y puede ser explicada por: (i) un efecto indirecto positivo del oxígeno a través de la exclusión de predadores y competidores (Levin et al. 1991, Neira et al. 2001b) y (ii) en términos de maximización del aprove- chamiento de la energía. Esto último lleva a una comunidad con una organización simple, poco madura en términos sucesionales constituida principalmente por organismos de pequeño tamaño corporal y con una alta tasa de recambio. Esta organización, como se verá más adelante, permite una rápida recuperación luego de eventos catastróficos (e.g., hipoxia severa). Durante períodos más benignos es posible capturar y procesar la energía del sistema en una forma más eficiente (Odum 1971, Pearson \& Rosenberg 1978, Díaz \& Rosenberg 1995).

\section{Producción secundaria y requerimientos meta- bólicos}

Si bien el modelo empírico propuesto por Tumbiolo \& Downing (1994) fue desarrollado originalmente para la macrofauna, las tasas de recambio $(\mathrm{P} / \mathrm{B})$ obtenidas en nuestro estudio $\left(10-18 \mathrm{a}^{-1}\right)$ concuerdan con los valores reportados por otros investigadores [media geométrica 16,4 e intervalo de confianza de $95 \%=11,0$ 24,5 $\mathrm{a}^{-1}$ (Vranke \& Heip 1986, Li et al. 1997, Alongi 1998)]. Estas tasas de recambio están relacionadas en forma proporcional al tamaño 
corporal y al tipo de desarrollo. Vranken \& Heip (1986) reportan razones P/B para algunas especies de nematodos, desde $1,5 \mathrm{a}^{-1}$ para especies de gran tamaño corporal (e.g., enóplido Pontonema vulgaris) a $69 \mathrm{a}^{-1}$ para especies de pequeño tamaño corporal y desarrollo rápido.

Los valores de producción secundaria meiobentónica estimados para la plataforma intermedia y externa [1,53 a 4,77 $\mathrm{gC} \mathrm{m}^{-2} \mathrm{a}^{-1}$ (Tabla $3)$ ], son comparables a los citados por Rowe (1985) para las zonas de surgencia del noroeste de África y Perú (4-5,8 $\left.\mathrm{gC} \mathrm{m}^{-2} \mathrm{a}^{-1}\right)$. La productividad $(\mathrm{P} / \mathrm{B})$ en estos sitios no presentó mayor variación estacional durante el período de estudio. En los sitios más someros, la productividad fue superior $\left(4,79\right.$ a $\left.32,19 \mathrm{gC} \mathrm{m}^{-2} \mathrm{a}^{-1}\right)$ a los reportados por el autor antes mencionado. Hacia mayo de 1998, estos valores presentaron una fuerte variación, triplicándose dentro de la bahía (Estación 4) y casi duplicándose en la plataforma interna (Estación 14). La boca de la bahía (Estación 7), si bien fue el sitio que presentó mayores valores de productividad, esta no tuvo una variación importante durante el período de estudio. Probablemente esto se deba a que en este sitio en años no-El Niño no se generan condiciones severamente anóxicas y reductoras, probablemente relacionado con sus mejores condiciones de circulación y ventilación del agua de fondo y por encontrarse a una profundidad por sobre el rango promedio de la ZMO. Dentro de la bahía, las condiciones de alta carga orgánica y estancamiento y en la plataforma, la incursión estival de la ZMO generan estacionalmente condiciones deficientes en oxígeno. Sin embargo, en estos últimos sitios entre mayo de 1997 y mayo de 1998 esto no se observó, además el sedimento superficial se mantuvo químicamente oxidado y no se acumularon altas concentraciones de ácido sulfhídrico en el agua intersticial (Gutiérrez 2000, Sellanes 2002), lo cual es consistente con los rápidos aumentos registrados en la abundancia y biomasa de la meiofauna hacia mayo de 1998 (Neira et al., 2001c). Por otra parte, en las zonas de mínimo oxígeno, donde se produce la combinación de condiciones hipóxicas con la presencia de grandes cantidades de materia orgánica lábil, se encuentra una fauna poco diversa pero con un alto flujo de energía y tasas de recambio (Díaz \& Rosenberg 1995). La boca de la bahía (Estación 7), conjuga entonces la ausencia de condiciones severamente deficientes en oxígeno con un alto flujo de materia orgánica de origen fitoplanctónico, potencialmente de alta calidad nutricional. Esto explica las extremadamente altas abundancias y biomasas presentes y los consecuentemente altos valores de producción secundaria, que en promedio son casi un orden de magnitud mayores que en los demás sitios. En este sitio, el valor estimado de la fracción de carbono orgánico que la meiofauna estaría reciclando, producción + respiración $\left(\mathrm{P}+\mathrm{R}_{\mathrm{t}}\right)$, se ubicaría entre los 65,49 y 104,28 g C $\mathrm{m}^{-2} \mathrm{a}^{-1}$. En tanto, para el interior de la bahía los valores fluctuaron entre 15,66 y $55,65 \mathrm{~g} \mathrm{C} \mathrm{m}^{-2}$ $\mathrm{a}^{-1}$, revelando un importante rol de la meiofauna en el flujo de energía. Esto es consistente con lo observado en otros ambientes con sedimentos orgánicamente enriquecidos (Duplisea \& Hargrave 1996).

Para obtener una idea del rol que podría estar jugando la meiofauna en el flujo total de energía en el ecosistema, se compararon los valores estimados de sus requerimientos metabólicos con los flujos de carbono orgánico $\left(\mathrm{C}_{\mathrm{org}}\right)$ anuales locales. Farías (1998) utilizando trampas de sedimentos, estimó los valores de los flujos de $\mathrm{C}_{\text {org }}$ entre $101,1 \pm 18,2$ (media $\pm \mathrm{DE}$ ) y $195,6 \pm 34,8 \mathrm{~g} \mathrm{C} \mathrm{m}^{-2} \mathrm{a}^{-1}$ para el interior y boca de la Bahía de Concepción, respectivamente. Al comparar estos valores con la producción de la meiofauna, se obtiene que, en promedio, entre un 36 y $45 \%$, respectivamente, de este $\mathrm{C}_{\text {org }}$ proveniente de la columna de agua podría estar siendo canalizado a través del meiobentos en estos sitios. Estas cifras son un poco mayores a las reportadas por Rudnick et al. (1985) para Bahía Narragansett (Rhode Island, USA) en sedimentos fangosos a $7 \mathrm{~m}$ de profundidad (27\%), pero en el rango descrito para la meiofauna (13 y $42 \%$ ) (Vanhove et al. 1997). En tanto, en la plataforma frente a Chile central existe escasa información sobre tasas de sedimentación. Sin embargo, recientemente Grunewald et al. (2002) señala para esta zona un valor de $228 \mathrm{~g} \mathrm{C} \mathrm{m}^{-2} \mathrm{a}^{-1}(100 \mathrm{~m})$. Estos antecedentes permiten estimar que aproximadamente un 3,0\% de este $\mathrm{C}_{\text {org }}$ podría estar siendo remineralizado o convertido a biomasa por el meiobentos en esta área.

Cabe señalar que las estimaciones realizadas en el presente estudio deben considerarse como una aproximación, debido principalmente a que los flujos de materia orgánica hacia el sedimento dependen principalmente de la producción primaria en la columna de agua (Graf 1992). En este sentido, en el área de estudio se han observado fuertes variaciones en los niveles de producción primaria a escala temporal y en particular durante la presencia de EN. En la zona central de Chile se han registrado valores de producción primaria que varían entre 1,5 a $5 \mathrm{~g}$ $\mathrm{C} \mathrm{m}{ }^{-2} \mathrm{~d}^{-1}$ durante períodos normales (no - El Niño), mientras que valores tan bajos como $0.17 \mathrm{~g} \mathrm{C} \mathrm{m}^{-2} \mathrm{~d}^{-1}$ fueron registrado durante $\mathrm{El}$ 
Niño 1997-1998 (L. Farías \& O. Ulloa, comunicación personal).

\section{Comparación con la macrofauna}

Como ya fuera indicado, en la mayoría de los ambientes marinos la macrofauna supera a la meiofauna en biomasa, sin embargo la razón $\mathrm{P} / \mathrm{B}$ de esta última es en general varias veces mayor (Higgins \& Thiel 1988, Giere 1993). De esto se desprende que a menor biomasa, el rol de la meiofauna puede llegar a ser similar e incluso superar al de la macrofauna en el flujo de energía a través del subsistema bentónico. En la Tabla 5 se comparan la biomasa de la macro- y meiofauna y sus correspondientes razones $\left(R_{b}\right)$ en distintos ambientes costeros y de plataforma. Los valores de biomasa de macrofauna encontrados durante EN en los sitios ubicados en el centro y boca de la bahía y plataforma interna de Concepción tanto para la macro- como para la meiofauna, están entre los mas altos reportados.
Para las comunidades macrobentónicas en el área de estudio, se puede considerar una razón $\mathrm{P} /$ B para la macrofauna de 4,5 $\mathrm{a}^{-1}$ (Carrasco \& Ar$\cos 1980)$ correspondiente a lo registrado para dos de las especies más dominantes en la plataforma frente a Concepción [e.g., el poliqueto Paraprionospio pinnata y el anfípodo Ampelisca araucana (Gallardo et al. 1995)]. Asumiendo entonces este valor como una estimación promedio para la macrofauna y el $\mathrm{P} / \mathrm{B}$ promedio calculado en este estudio para la meiofauna frente a Concepción $\left(13,6 \mathrm{a}^{-1}\right)$, se obtiene que para que la macro y meiofauna tengan un rol similar en los requerimientos energéticos su $R_{b}$ debe ubicarse en torno a tres (i.e., 13,6 / 4,5). Esta razón es similar a la reportada por Rowe (1985) para el margen peruano $\left(\sim 15^{\circ} \mathrm{S}\right)$ y a la razón general de 5:1 propuesta por Gerlach (1971).

Frente a Concepción, la biomasa de la macrofauna en el mismo período de estudio fue entre 4,3 y 48,8 veces superior a la de la meiofauna (Fig. 4).

TABLA 5

Comparación entre las biomasas de la macro- y meiofauna de la zona de plataforma en distintas localidades. $\mathrm{R}_{\mathrm{bo}}$ es la razón entre la biomasa de la macrofauna y la de la meiofauna.

Modificado de Le Guellec \& Bodin (1992)

Comparison among continental shelf macro- and meiofaunal biomasses in different localities. $\mathrm{R}_{\mathrm{b}}$ is the ratio between macrofaunal and meiofaunal biomass. Modified from Le Guellec \& Bodin (1992)

\begin{tabular}{|c|c|c|c|c|c|}
\hline \multirow[t]{2}{*}{ Lugar } & \multirow{2}{*}{$\begin{array}{l}\text { Profundidad } \\
\text { (m) }\end{array}$} & \multicolumn{2}{|c|}{$\begin{array}{c}\text { Biomasa } \\
\text { (media o rango; } \\
\mathrm{gm}^{-2} \mathrm{PS} * \text { ) }\end{array}$} & \multirow[t]{2}{*}{$\mathrm{R}_{\mathrm{b}}$} & \multirow[t]{2}{*}{ Referencia } \\
\hline & & Macrofauna & Meiofauna & & \\
\hline Göta river estuary, Mar del Norte & $3-50$ & $0,7-15,4$ & $0,3-0,8$ & $2,8-19,2$ & Nyholm et al. (1977) \\
\hline Mar del Norte & Sin datos & $1,7-2,2$ & $0,6-1,9$ & $1,1-2,8$ & Govaere et al. (1980) \\
\hline Banyuls, Mar Mediterráneo & $14-87$ & 1,9 & $0,1-0,5$ & 6,8 & Guille \& Soyer (1971) \\
\hline Helgoland Bight, Mar del Norte & $16-49$ & $1,8-12,2$ & $0,05-0,4$ & $24-90$ & Stripp (1969) \\
\hline Helgoland Bight, Mar del Norte & 35 & 7,4 & 0,6 & 12,6 & Gerlach (1978) \\
\hline Askö-Landsort, Mar Báltico & $9-50$ & 7,8 & 1,0 & 7,8 & Ankar \& Elmgren (1976) \\
\hline Bothnian Bay, Mar Báltico & $5-220$ & 0,15 & 0,3 & 0,5 & Elmgren (1980) \\
\hline Bothnian Sea, Mar Báltico & $5-100$ & 10,6 & 1,1 & 9,7 & Elmgren (1980) \\
\hline Gullmar Fjord, Suecia & $42-120$ & 3 & 0,4 & 7,5 & Evans (1983) \\
\hline Fladen Ground, Mar del Norte & 150 & 3,1 & 0,25 & 12,4 & De Wilde et al. (1986) \\
\hline South Is., N. Zelandia, O. Pacífico & 200 & 6,1 & 0,6 & 10,2 & Probert (1986) \\
\hline Morlaix Bay, Canal de la Mancha & Sin datos & 4,6 & 0,4 & 11,5 & Dauvin (1985) \\
\hline Douarnenez Bay, Francia & $0-35$ & $3,4-12,8$ & $0,9-2,6$ & $1,4-5,6$ & Bodin et al. (1985) \\
\hline St. Brieuc, Canal de la Mancha & $2-13$ & 9,3 & $0,5-1,1$ & $8,5-19$ & Le Guellec \& Bodin (1992) \\
\hline Goban Spur, Atlántico Norte & 208 & 1,37 & 0,17 & 8 & Flach et al. (1999) \\
\hline Magen de Perú central & $63-110$ & 13,8 & 3,8 & 3,6 & Rowe (1985) \\
\hline Bahía de Concepción, Chile (centro) & 27 & $1,9-54,3$ & $0,4-1,5$ & $4,3-36,7$ & Este estudio \\
\hline Bahía de Concepción, Chile (boca) & 34 & $15,9-40,4$ & $2,2-3,2$ & $7,1-12,4$ & Este estudio \\
\hline Plataforma interna, Chile & 64 & $11,1-38,8$ & $0,8-1,5$ & $7,6-48,8$ & Este estudio \\
\hline Plataforma intermedia, Chile & 88 & $4,2-16,9$ & $0,5-0,7$ & $8,5-25,5$ & Este estudio \\
\hline Plataforma externa, Chile & 120 & $7,2-12,7$ & $0,2-0,4$ & $18,9-46,0$ & Este estudio \\
\hline
\end{tabular}

$* \mathrm{PS}=$ Peso seco 


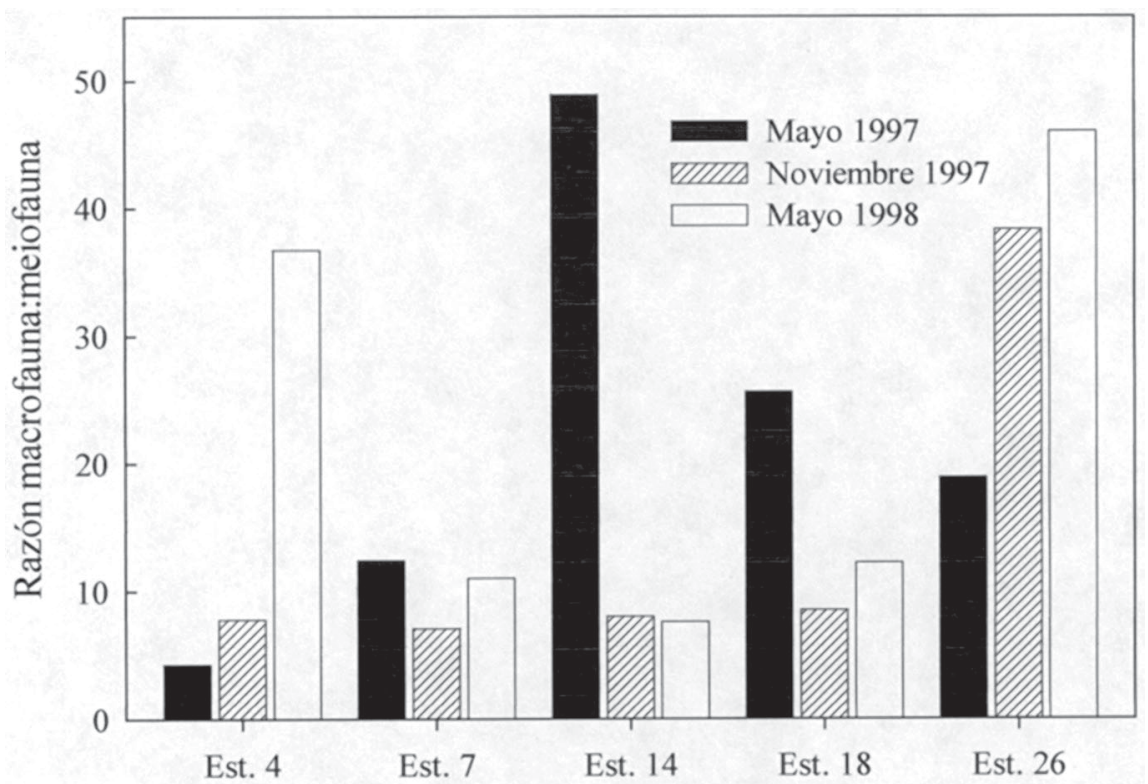

Fig. 4: Razones macrofauna: meiofauna $\left(\mathrm{R}_{\mathrm{b}}\right)$ en relación a la biomasa para los meses de mayo y noviembre de 1997 y mayo de 1998, para los distintos sitios.

Macrofauna: meiofauna biomass ratios $\left(\mathrm{R}_{\mathrm{b}}\right)$ for May and November 1997 and May 1998.

Los menores valores correspondieron al interior de la bahía en mayo de 1997, sugiriendo un rol similar de la meiofauna respecto al de la macrofauna en la producción secundaria (Fig. 4). Sin embargo, hacia mayo de 1998 , esta importancia disminuyó, debido al desproporcionado aumento en la biomasa de la macrofauna $\left(\mathrm{R}_{\mathrm{b}}\right.$ $=36,7)$ relacionado al parecer con las mejores condiciones ambientales (Gutiérrez et al. 2000). En la plataforma interna (Estación 14) durante EN, debido al aumento de la meiofauna en relación a la macrofauna, el rol del meiobentos aumentó considerablemente (Fig. 4). Tanto en la boca de la bahía (Estación 7) como en la plataforma intermedia estas variaciones no fueron tan evidentes mientras que en la paltaforama externa (Estación 26) el rol de la meiofauna disminuyó considerablemente (Fig. 4).

En síntesis, frente a Concepción, la meiofauna juega un importante rol en el flujo de la energía a través del subsistema bentónico, con una capacidad potencial de utilizar y/o reciclar gran parte del $\mathrm{C}_{\text {org }}$ que llega a los sedimentos. Comparando su biomasa con la de la macrofauna y considerando las mayores tasas de generación de la meiofauna, esta última puede llegar a tener un papel incluso similar al de la macrofauna en el flujo de energía del sistema. Este papel varía entre localidades tanto en importancia como en la magnitud del efecto que sobre él tiene el ciclo ENOS.

\section{AGRADECIMIENTOS}

Nuestro agradecimiento al capitán y tripulación de la L/C Kay Kay, al personal auxiliar de la Estación de Biología Marina de Dichato, colegas que colaboraron tanto en la recolección de muestras como en los análisis de laboratorio y a la Dra. Lisa Levin (SCRIPPS) por sus comentarios. El Servicio de Intercambio Académico Alemán (DAAD), la Escuela de Graduados de la Universidad de Concepción y el Consejo Nacional de Investigación Ciencia y Tecnología (CONICYT) prestaron apoyo financiero a JS durante sus estudios de postgrado en la Universidad de Concepción. Extendemos nuestro agradecimiento a CONICYT en el marco de las becas de apoyo para la realización de tesis doctoral otorgadas a JS y EQ. Los proyectos FONDECYT No 1971336 y FONDAP Humboldt y COPAS, dirigidos por el Prof. Dr. Víctor A. Gallardo, apoyaron las distintas fases de ejecución de este trabajo.

\section{LITERATURA CITADA}

ALLER RC \& JY ALLER (1992) Meiofauna and solute transport in marine muds. Limnology and Oceanography 37: 1018-1033.

ALLER JY, RC ALLER \& MA GREEN (2002) Benthic faunal assemblages and carbon supply along the continental shelf/shelf break-slope off Cape 
Hatteras, North Carolina. Deep-Sea Research II 49: 4599-4625.

ALONGI DM (1998) Coastal ecosystem processes. CRC Press, Boca Raton, Florida, USA. 419 pp.

ANDRASSY I (1956) Die Rauminhalts-und Gewichts bestimmung der Fadenwürmer (Nematoden). Acta Zoologica Academica Scientiarum Hungaricae 2: 1-15.

ANKAR S \& R ELMGREN (1976) The benthic macroand meiofauna of the Askö-Landsort area (northern Baltic proper), a stratified random sampling survey. Contributions of the Askö Laboratory (University of Stockholm) 11: 1-115.

BANSE K (1979) On weight dependence of net growth efficiency and specific respiration rates among field populations of invertebrates. Oecologia 38: 111-126.

BANSE K \& S MOSHER (1980) Adult body mass and annual production/biomass relationships of field populations. Ecological Monographs 50: 355-379.

BARNETT PR, J WATSON J \& D CONNELLY (1984) A multiple corer for taking virtually undisturbed samples from shelf, bathyal and abyssal sediments. Oceanologica Acta 7: 399-408.

BODIN P (1985) Les peuplements de copépodes harpacticoides (Crustacea) des sédiments meubles de la zone intertidale des côtes charentaises (Atlantique). Mémories du Museé Nationale d'Historie Naturelle (France) 104: 1-120.

BREY T (2001) Population dynamics in benthic invertebrates. A virtual handbook. Version 01.2. http://www.awi-bremerhaven.de/Benthic/ Ecosystem/FoodWeb/Handbook

CARRASCO FD \& DF ARCOS (1980) Estimación de la producción secundaria de Paraprionospio pinnata (Spionidae) frente a la Bahía de Concepción, Chile. Boletim do Instituto Oceanografico de Sao Paulo (Brasil) 29: 79-82.

COOK AA, PJD LAMBSHEAD, LE HAWKINS, N MITCHELL \& LA LEVIN (2000) Nematode abundance at the oxygen minimum zone in the Arabian Sea. Deep-Sea Research II 47: 75-85

COULL BC (1988) Ecology of the marine meiofauna. En: Higgins RP \& $\mathrm{H}$ Thiel (eds) Introduction to the study of meiofauna: 18-38. Smithsonian Institution Press, Washington, District of Columbia, USA.

COULL BC (1990) Are members of the meiofauna food for higher trophic levels? Transactions of the American Microscopical Society 109: 233-246.

COULL BC (1999) Role of meiofauna in estuarine softbottom habitats. Australian Journal of Ecology 24: 327-343.

COULL BC, JG GREENWOOD, DR FIELDER \& BA COULL (1995) Subtropical Australian juvenile fish eat meiofauna: experiments with winter whiting (Sillago maculata) and observations on other species. Marine Ecology Progress Series 125: 13-19.

DAUVIN JC (1985) Dynamics of an Abra prismatica population (Mollusca, Bivalvia) from the Bay of Morlaix (western English Channel). Annales de 1'Institut Oceanographique, Nouvelle Serie (France) 62: 1-12.

DE BOVÉE F, POJ HALL, S HULTH, G HULTHE, A LANDÉN \& A TENGBERG (1996) Quantitative distribution of metazoan meiofauna in continental margin sediments of the Skagerrak (Northeastern North Sea). Journal of Sea Research 35: 189-197.

DE WILDE PAWJ, EM BERGUIS \& A KOK (1986) Biomass and activity of benthic fauna in the Fladen Ground (northern North Sea). Netherlands Journal of Sea Research 20: 313-323.

DÍAZ RJ \& R ROSENBERG (1995) Marine benthic hypoxia: a review of its ecological effects and the behavioural responses of benthic macrofauna. Oceanography and Marine Biology Annual Revue 33: 245-303

DUPLISEA DE \& BT HARGRAVE (1996) Response of meiobenthic size-structure, biomass and respiration to sediment organic enrichment. Hydrobiologia 339: 161-170.

ELMGREN R (1980) Structure and dynamics of Baltic benthos communities, with particular reference to the relationship between macro- and meiofauna. Kieler Meeresforschung 4: 1-22.

EVANS S (1983) Production, predation and food. Niche segregation in a marine shallow soft-bottom community. Marine Ecology Progress Series 10: 147-157.

FARÍAS L (1998) Destino del nitrógeno orgánico en sedimentos de una bahía eutroficaza: reciclaje vs. acumulación. Tesis de Doctorado en Oceanografía, Universidad de Concepción, Concepción, Chile. 157 pp.

FELLER RJ \& RM WARWICK (1988) Energetic. En: Higgins RP \& H Thiel (eds) Introduction to the study of meiofauna: 181-196. Smithsonian Institution Press, Washington, District of Columbia, USA.

FINDLAY SEG \& KR TENORE (1982) Effect of a free living marine nematode (Dipolaimella chitwoodi) on detrital carbon mineralization. Marine Ecology Progress Series 8: 161-166.

FLACH E, J VANAVERBEKE \& C HEIP (1999) The meiofauna:macrofauna ratio across the continental slope of the Goban Spur (north-east Atlantic). Journal of the Marine Biology Association UK 79: 233-241.

FOSSING H, VA GALLARDO, BB JØRGENSEN, M HÜTTEL, LP NIELSEN, H SCHULZ, DE CANFIELD, S FORSTER, RN GLUD, JK GUNDERSEN, J KÜVER, NB RAMSING, A TESKE, B THAMDRUP \& O ULLOA (1995) Concentration and transport of nitrate by the matforming sulphur bacterium Thioploca. Nature 374: 713-715.

GALLARDO VA (1985) Efectos del fenómeno de El Niño sobre el bentos sublitoral frente a Concepción, Chile. En: Arntz W, A Landa \& J Tarazona (eds) El Niño y su impacto en la fauna marina: 79-85. Instituto del Mar del Perú-Callao, Boletín Extraordinario $\mathrm{N}^{\circ} 198$

GALLARDO VA, FD CARRASCO, R ROA \& JI CAÑETE (1995) Ecological patterns in the benthic macrobiota across the continental shelf off central Chile. Ophelia 40: 167-188.

GERLACH SA (1971) On the importance of marine meiofauna for benthos communities. Oecologia 6: 176-190.

GERLACH SA (1978) Food-chain relationships in subtidal silty sand marine sediments and the role of the meiofauna in stimulating bacterial productivity. Oecologia 33: 55-69.

GIERE O (1993) Meiobenthology. The microscopic fauna in aquatic sediments. Springer-Verlag, Berlin, Germany. 328 pp.

GOVAERE J, D VAN DAMME, C HEIP \& L DE CONINCK (1980) Benthic communities in the southern bight of the North Sea and their use in ecological monitoring. Helgoländer Meeresuntersuchungen 33: 507-521.

GRAF G (1992) Benthic-pelagic coupling: a benthic view. Oceanography Marine Biology Annual Review 30: 149-190.

GRUNEWALD A, C MORALES, H GONZALES, C SYLVESTER \& R CASTRO (2002) Grazing impact of copepod assemblages and gravitational flux in coastal and oceanic waters off central Chile during 
two contrasting seasons. Journal of Plankton Research 24: 55-67.

GUILLE A \& J SOYER (1971) Contribution à l'étude comparée des biomasses du macrobenthos et du méiobenthos de substrat meuble au large de Banyuls-sur-Mer. Vie et Milieu 22: 15-29.

GUTIÉRREZ D (2000) Bioperturbación y macrofauna en fondos sublitorales de un área de surgencias frente a Chile central (36 $\left.30^{\circ} \mathrm{S}\right)$ : variación espacial y temporal en el período 1997-1998. Tesis de Doctorado en Oceanografía. Universidad de Concepción, Concepción, Chile. 221 pp.

GUTIÉRREZ D, VA GALLARDO, S MAYOR, C NEIRA, C VÁSQUEZ, J SELLANES, M RIVAS, A SOTO, FD CARRASCO \& M BALTAZAR (2000) Effects of dissolved oxygen and fresh organic matter on macrofaunal bioturbation potential in sublittoral bottoms off central Chile, during the 1997-98 El Niño. Marine Ecology Progress Series 202: 81-99.

HIGGINS RP \& H THIEL (1988) Introduction to the study of meiofauna. Smithsonian Institution Press, Washington, District of Columbia, USA. 488 pp.

JOSEFSON AB \& B WIDBOM (1988) Differential response of benthic macrofauna and meiofauna to hypoxia in the Gullmar Fjord basin. Marine Biology 100: 31-44.

KANE RP (1999) Some characteristics and precipitation effects of the El Niño 1997-1998. Journal of Atmospheric and Solar-Terrestrial Physics 61: 1325-1346.

LE GUELLEC C \& P BODIN (1992) Meiobenthos of the Bay of Saint-Brieuc (North Brittany, France). I: Quantitative distribution in subtidal and intertidal zones. Oceanologica Acta 15: 661-671.

LI J, M VINCX \& PMJ HERMAN (1997) Carbon flows through meiobenthic nematodes in the Westerschelde Estuary. Fundamentals of Applied Nematology 20: 487-494.

MC PHADEN MJ (1999) Genesis and evolution of the 1997-98 El Niño. Science 283: 950-954.

MIRTO S, T LA ROSA, R DANOVARO \& A MAZZOLA (2000) Microbial and meiofauna response to intensive mussel-farm biodeposition in coastal sediments of the western Mediterranean. Marine Pollution Bulletin 40: 244-252.

MURRELL MC \& JW FLEEGER (1989) Meiofauna abundance on the Gulf of Mexico continental shelf affected by hypoxia. Continental Shelf Research 9: 1049-1062.

MURPHY RC (1926) Oceanic and climatic phenomena along the west coast of South America during 1925. Geographical Revue 16: 25-54.

NEIRA, C \& T HÖPNER (1994) The role of Heteromastus filiformis (Capitellidae, Polychaeta) in organic carbon cycling. Ophelia 39: 55-73.

NEIRA C, G GAD, NL ARROYO \& W DECRAEMER (2001a) Glochinema bathyperuvensis sp. n. (Nematoda, Epsilonematidae): a new species from Peruvian bathyal sediments, SE Pacific Ocean. Contributions to Zoology 70: 147-159.

NEIRA C, J SELLANES, LA LEVIN, WE ARNTZ (2001b) Meiofaunal distributions on the Peru margin: relationship to oxygen and organic matter availability. Deep-Sea Research I 48: 2453-2472.

NEIRA C, J SELLANES, A SOTO, D GUTIÉRREZ, VA GALLARDO (2001c) Meiofauna and sedimentary organic matter off central Chile: response to changes caused by the 1997-1998 El Niño. Oceanologica Acta 24: 313-328.

NYHOLM KG \& I OLSON (1973) Seasonal fluctuations of the meiobenthos in an estuary on the Swedish west coast. Zoon 1: 1-12.
NYHOLM KG, I OLSON \& L ANDREN (1977) Quantitative investigations on the macro-and meiobenthic fauna in the Göta river estuary. Zoon 5: $15-28$

ODUM HT (1971) Environment, power and society. Wiley-Interscience, New York, New York, USA. $331 \mathrm{pp}$

PEARSON TH \& ROSENBERG R (1986) Feast and famine: structuring factors in marine benthic communities. En: Gee JHR \& PS Giller (eds) Organization of communities: past and present: 373-395. Blackwell Scientific Publications, London, United Kingdom.

PROBERT PK (1986) Energy transfer through the shelf benthos off the west coast of South Island, new Zealand. New Zealand Journal of Marine and Freshwater Research 20: 407-417.

RADZIEJEWSKA T, JW FLEEGER, N RABALAIS \& KR CARMAN (1996) Meiofauna and sediment chloroplastic pigments on the continental shelf off Louisiana, USA. Continental Shelf Research 16: 1699-1723.

RAINER SF \& RC FITZHARDINGE (1981) Benthic communities in an estuary with periodic deoxygenation. Australian Journal of Marine and Freshwater Research 32: 227-243.

RICCIARDI A \& E BOURGET (1998) Weight-to-weight conversion factors for marine benthic macroinvertebrates. Marine Ecology Progress Series 163: 245-251.

RIEMANN F (1988) Nematoda. En: Higgins RP \& H Thiel (eds) Introduction to the study of meiofauna: 293301. Smithsonian Institution Press, Washington, District of Columbia, USA.

ROWE G (1985) Benthic production and processes off Baja California, northwest Africa and Peru: a classification of benthic subsystem in upwelling ecosystem. Simposio internacional sobre las áreas de afloramiento mas importantes del oeste africano (Cabo Blanco y Benguela): 589-612. Instituto de investigaciones pesqueras, Barcelona, España, Volumen II.

ROWE G, G BOLAND, W PHOEL, R ANDERSON \& P BISCAYE (1994) Deep-sea floor respiration as an indication of lateral input of biogenic detritus from continental margins. Deep-Sea Research II 41: 657-668.

RUDNICK DT, R ELMGREN \& JB FRITHSEN (1985) Meiofaunal prominence and benthic seasonality in a coastal marine ecosystem. Oecologia 67: 157-168.

SCHWINGHAMER P, B HARDGRAVE, D PEER \& C HAWKINS (1986) Partitioning of production and respiration among size groups of organisms in an intertidal benthic community. Marine Ecology Progress Series 31: 131-142.

SELLANES J (2002) Efectos de El Niño 1997-98 sobre los indicadores bioquímicos de la calidad de la materia orgánica sedimentaria y la meiofauna en un área de surgencia costera de Chile central. Tesis de doctorado en oceanografía, Universidad de Concepción, Concepción, Chile. $x+192$ pp.

SNEATH PHA \& RR SOKAL (1973) Numerical taxonomy. W. H. Freeman \& Co., San Francisco, California, USA. 573 pp.

STREET GT, BC COULL, GT CHANDLER \& DM SANGER (1998) Predation on meiofauna by juvenile spot Leiostomus xanthurus (Pisces) in contaminated sediments from Charleston Harbor, South Carolina, USA. Marine Ecology Progress Series 170: 261-268.

STRIPP K (1969) Das Verhältnis von Makrofauna und Meiofauna in den Sedimenten der Helgoländer 
Bucht. Veröffentlichungen des Instituts Meeresforschung Bremerhaven 12: 143-148.

TENORE KR (1977) Food chain pathways in detrital feeding benthic communities: a review, with new observations on sediment resuspension and detrital recycling. En: Coull BC (ed) Ecology of marine benthos: 37-53. University of South Carolina Press, Columbia, South Carolina, USA.

THIEL H (1978) Benthos in upwelling regions. En: Boje R \& M Tomczak (eds) Upwelling ecosystems: 124138. Springer, Berlin, Germany.

TUMBIOLO M \& JA DOWNING (1994) An empirical model for the prediction of secondary production in marine benthic invertebrate populations. Marine Ecology Progress Series 114: 165-174.

VANHOVE S, J WITTOECK, M BEGHYN, D VAN GANSBEKE, A VAN KENHOVE, A COOMANS
\& M VINCX (1997) Role of the meiobenthos in Antarctic ecosytems. En: Caschetto S (ed) Belgian research programme on the Antarctic: scientific results of phase III (1992-1996), Volume I: 1-59. Federal Office for Scientific, Technical and Cultural Affairs, Brussel, Belgium.

VRANKEN G \& C HEIP (1986) The productivity of marine nematodes. Ophelia 26: 429-442.

WELLS JBJ (1988) Copepoda. En: Higgins RP \& H Thiel (eds) Introduction to the study of meiofauna: 380388. Smithsonian Institution Press, Washington, District of Columbia, USA.

WIESER W (1960) Benthic studies in Buzzards Bay. II. The meiofauna. Limnology and Oceanography 5: 121-137.

ZAR JH (1974) Biostatistical analysis. Prentice Hall Inc., Englewood Cliffs, New Jersey, USA. 620 pp.

Editor Asociado: Sergio Navarrete

Recibido el 21 de agosto de 2002; aceptado el 13 de mayo de 2003 